\title{
Statistics of the von Mises stress response for structures subjected to random excitations
}

\author{
Mu-Tsang Chen ${ }^{\mathrm{a}, *}$ and Ronald Harichandran ${ }^{\mathrm{b}}$ \\ aANYS Inc., 275 Technology Dr., Canonsburg, \\ PA 15317, USA \\ ${ }^{\mathrm{b}}$ Department of Civil and Environmental \\ Engineering, Michigan State University, \\ East Lansing, MI 48824, USA
}

Finite element-based random vibration analysis is increasingly used in computer aided engineering software for computing statistics (e.g., root-mean-square value) of structural responses such as displacements, stresses and strains. However, these statistics can often be computed only for Cartesian responses. For the design of metal structures, a failure criterion based on an equivalent stress response, commonly known as the von Mises stress, is more appropriate and often used. This paper presents an approach for computing the statistics of the von Mises stress response for structures subjected to random excitations. Random vibration analysis is first performed to compute covariance matrices of Cartesian stress responses. Monte Carlo simulation is then used to perform scatter and failure analyses using the von Mises stress response.

Keywords: Random vibration, von Mises stress, Monte Carlo simulation, covariance matrix, failure analysis

\section{Introduction}

Many structural and mechanical systems are exposed to stochastic loads, e.g., aircrafts subjected to atmospheric turbulence, buildings and bridges subjected to earthquakes, vehicle components subjected to vibrations arising from rough roads, and ships and offshore platforms subjected to wind and wave loads. The theory of random vibration is central to the analysis and design of structures exposed to random

*Corresponding author: Tel.: +1 724514 3105; Fax: +1 724 514 3118; E-mail: mtc@ansys.com. excitations and is being increasingly used in practice for a variety of engineering problems (Chen [4]; Nigam [12]; Soong and Grigoriu [13]). In recent years, RVA in conjunction with the finite element (FE) method is available in several commercial software, such as ANSYS, NASTRAN, ABAQUS, I-DEAS, ALGOR and ADINA. Most of these software can be used to compute root-mean-square (r.m.s.) values of Cartesian displacements, stresses and strains. However, Cartesian responses alone are often inadequate for failure analyses in structural design. A failure criterion based on the maximum octahedral shear stress, often referred to as the von Mises theory, is usually used. This theory is widely regarded to be the most reliable for the design of ductile materials. The statistics of the von Mises stress cannot be readily computed in FE analysis software because it is nonlinearly related to the Cartesian stresses and its statistics cannot be easily related to those of the Cartesian stresses. Harichandran and Chen [7] explored the use of a first-order second-moment approach to approximately compute statistics of principal stress-related quantities, but calibrated correction factors were needed to obtain acceptable accuracy and the method may not be sufficiently robust.

In this paper, direct Monte Carlo simulation (MCS) is used to compute the statistical moments of the von Mises stress. Because an explicit formula relating the von Mises stress to the Cartesian stresses is available, the simulation requires little computer time. For 3D problems, correlations between the Cartesian stresses at each node must be taken into account, and hence the twenty-one components of a $6 \times 6$ variancecovariance matrix at each node must be computed from RVA prior to the MCS. Using closed-form solutions for stochastic responses from RVA (Chen and Ali [5]; Harichandran [6]) the variance-covariance matrix at each node can be computed quickly and efficiently for common types of excitations. As a result, computation of the statistics of the von Mises 
stress, and other quantities such as the stress intensity and maximum shear stress, becomes feasible. Scatter analysis based on the von Mises stress response of a structure subjected to random vibration is also presented in this study. Histograms approximating the probability distribution function (PDF) and cumulative distribution function $(\mathrm{CDF})$ of the von Mises stress can be obtained, which can provide useful information for designing against yield and fatigue failures.

In failure analysis, a design strength taken as a deterministic value or a random variable (typically Gaussian or Weibull) may be specified such that probability of failure of a structure can be computed. The failure probability estimated by MCS is approximate, but the coefficient of variation of the failure probability also can be estimated to assess its accuracy. In addition, the required strength for engineering design corresponding to a desired probability of failure may be estimated easily through MCS. In this paper, a simple example consisting of a shaker table excited by random loads, is used to illustrate the various features of the analysis. By using the proposed technique, the von Mises failure criterion is applied for the reliability-based design of the shaker table.

\section{Finite element-based RVA}

The dynamic equations of motion of a finite element system may be expressed in the partitioned form (Harichandran and Wang [8]):

$$
\begin{aligned}
& {\left[\begin{array}{c}
M_{\mathrm{FF}} M_{\mathrm{FR}} \\
M_{\mathrm{RF}} M_{\mathrm{RR}}
\end{array}\right]\left[\begin{array}{c}
\left\{\ddot{u}_{\mathrm{F}}\right\} \\
\left\{\ddot{u}_{\mathrm{R}}\right\}
\end{array}\right]+\left[\begin{array}{c}
C_{\mathrm{FF}} C_{\mathrm{FR}} \\
C_{\mathrm{RF}} C_{\mathrm{RR}}
\end{array}\right]\left[\begin{array}{l}
\left\{\dot{u}_{\mathrm{F}}\right\} \\
\left\{\dot{u}_{\mathrm{R}}\right\}
\end{array}\right]} \\
& +\left[\begin{array}{ll}
K_{\mathrm{FF}} & K_{\mathrm{FR}} \\
K_{\mathrm{RF}} & K_{\mathrm{RR}}
\end{array}\right]\left[\begin{array}{l}
\left\{u_{\mathrm{F}}\right\} \\
\left\{u_{\mathrm{R}}\right\}
\end{array}\right]=\left[\begin{array}{l}
\{P\} \\
\{R\}
\end{array}\right]
\end{aligned}
$$

in which $\left\{u_{\mathrm{F}}\right\}$ are free degrees of freedom (DOFs), $\left\{u_{\mathrm{R}}\right\}$ are restrained DOFs, and $\{P\}$ and $\{R\}$ are the excitations at free nodes and reactions at the restrained nodes, respectively. The excitations $\{P\}$ are assumed to be zero-mean stationary stochastic processes. The free nodal displacement can be decomposed into pseudo-static and dynamic parts as $\left\{u_{\mathrm{F}}\right\}=\left\{u_{\mathrm{s}}\right\}+\left\{u_{\mathrm{d}}\right\}$. Assuming light damping and performing modal analysis and RVA, the mean-square displacement response can be expressed as

$$
\sigma_{u_{\mathrm{F}}}^{2}=\sigma_{u_{\mathrm{s}}}^{2}+\sigma_{u_{\mathrm{d}}}^{2}+2 \operatorname{Cov}\left(u_{\mathrm{s}}, u_{\mathrm{d}}\right)
$$

where the mean-square values of the $i$-th dynamic and pseudo-static displacements and their covariance can be respectively obtained as

$$
\begin{aligned}
& \sigma_{u_{\mathrm{d}_{i}}}^{2}=\sum_{j=1}^{n} \sum_{k=1}^{n} \Phi_{i j} \Phi_{i k}\left[\sum_{l=1}^{t} \sum_{m=1}^{t} \frac{\Phi_{l j} \Phi_{m k}}{M_{j} M_{k}}\right. \\
& \quad \times \int_{0}^{\infty} \overline{H_{j}(\omega)} H_{k}(\omega) S_{P_{l} P_{m}}(\omega) \mathrm{d} \omega \\
& \quad+\sum_{l=1}^{r} \sum_{m=1}^{r} \Gamma_{l j} \Gamma_{m k} \\
& \left.\quad \times \int_{0}^{\infty} \overline{H_{j}(\omega)} H_{k}(\omega) S_{\ddot{u}_{\mathrm{R}_{l}} \ddot{\mathrm{R}}_{\mathrm{R}}}(\omega) \mathrm{d} \omega\right], \\
& \sigma_{u_{\mathrm{s}_{i}}}^{2}=\sum_{l=1}^{r} \sum_{m=1}^{r} A_{i l} A_{i m} \int_{0}^{\infty} \omega^{-4} S_{\ddot{u}_{\mathrm{R}_{l}} \ddot{u}_{\mathrm{R}_{m}}}(\omega) \mathrm{d} \omega
\end{aligned}
$$

and

$$
\begin{gathered}
\operatorname{Cov}\left(u_{\mathrm{s}_{i}}, u_{\mathrm{d}_{i}}\right)=-\sum_{j=1}^{n} \sum_{l=1}^{r} \sum_{m=1}^{r} \Phi_{i j} A_{i l} \Gamma_{m j} \\
\times \int_{0}^{\infty} \omega^{-2} H_{j}(\omega) S_{\ddot{u}_{\mathrm{R}_{l}} \ddot{u}_{\mathrm{R}_{m}}}(\omega) \mathrm{d} \omega
\end{gathered}
$$

where

- $n=$ number of modes, $t=$ number of free DOF with force excitations, and $r=$ number of restrained DOF with acceleration excitations.

- $\Phi_{l j}=l j$-th element of the displacement mode shape matrix, $\Gamma_{l j}=l$-th element of the participation vector for mode $j, A_{i l}=$ the $i$-th displacement response due to a unit displacement at restrained DOF $l$.

- $S_{P_{l} P_{m}}(\omega)$ and $S_{\ddot{u}_{\mathrm{R}}} \ddot{u}_{\mathrm{R}_{m}}(\omega)=$ power spectral density (PSD) functions of nodal force and base acceleration excitations, respectively; $\omega=$ circular frequency.

- $H_{j}(\omega)=j$-th modal frequency response function given by

$$
H_{j}(\omega)=\frac{1}{\omega_{j}^{2}-\omega^{2}+2 \mathrm{i} \omega_{j} \zeta_{j} \omega},
$$

where $\omega_{j}$ and $\zeta_{j}$ are the modal frequency and damping ratio. An overbar denotes the complex conjugate.

\section{Cartesian stress responses}

As with the displacement response, any Cartesian stress can also be decomposed into pseudo-static and 
dynamic parts, $\left\{\tau_{\mathrm{s}}\right\}$ and $\left\{\tau_{\mathrm{d}}\right\}$, respectively. Consider two Cartesian stresses, $\tau_{1}$ and $\tau_{2}$, which can be expressed as $\tau_{1}=\tau_{1 \mathrm{~s}}+\tau_{1 \mathrm{~d}}$ and $\tau_{2}=\tau_{2 \mathrm{~s}}+\tau_{2 \mathrm{~d}}$, respectively. Thus,

$$
\begin{aligned}
& E\left[\tau_{1}, \tau_{2}\right]=E\left[\left(\tau_{1 \mathrm{~s}}+\tau_{1 \mathrm{~d}}\right)\left(\tau_{2 \mathrm{~s}}+\tau_{2 \mathrm{~d}}\right)\right] \\
& =E\left[\tau_{1 \mathrm{~d}} \tau_{2 \mathrm{~d}}+\tau_{1 \mathrm{~s}} \tau_{2 \mathrm{~s}}+\tau_{1 \mathrm{~d}} \tau_{2 \mathrm{~s}}+\tau_{1 \mathrm{~s}} \tau_{2 \mathrm{~d}}\right],
\end{aligned}
$$

where $E[\cdot]$ represents the expected value. Because all Cartesian stresses are assumed to be zero-mean Gaussian processes, Eq. (7) becomes

$$
\begin{aligned}
\operatorname{Cov}\left(\tau_{1}, \tau_{2}\right)= & \operatorname{Cov}\left(\tau_{1 \mathrm{~d}}, \tau_{2 \mathrm{~d}}\right)+\operatorname{Cov}\left(\tau_{1 \mathrm{~s}}, \tau_{2 \mathrm{~s}}\right) \\
& +\operatorname{Cov}\left(\tau_{1 \mathrm{~d}}, \tau_{2 \mathrm{~s}}\right)+\operatorname{Cov}\left(\tau_{1 \mathrm{~s}}, \tau_{2 \mathrm{~d}}\right) .
\end{aligned}
$$

As a result, the covariance matrix of the six Cartesian stresses at the $i$-th node may be expressed as

$$
\begin{aligned}
& {\left[\operatorname{Cov}\left(\tau_{p}, \tau_{q}\right)\right]_{i}=\left[\operatorname{Cov}\left(\tau_{p \mathrm{~d}}, \tau_{q \mathrm{~d}}\right)\right]_{i}+\left[\operatorname{Cov}\left(\tau_{p \mathrm{~s}}, \tau_{q \mathrm{~s}}\right)\right]_{i}} \\
& +\left[\operatorname{Cov}\left(\tau_{p \mathrm{~d}}, \tau_{q \mathrm{~s}}\right)\right]_{i}+\left[\operatorname{Cov}\left(\tau_{p \mathrm{~s}}, \tau_{q \mathrm{~d}}\right)\right]_{i},
\end{aligned}
$$

in which $\left[\operatorname{Cov}\left(\tau_{p \mathrm{~s}}, \tau_{q \mathrm{~s}}\right)\right]_{i}$ and $\left[\operatorname{Cov}\left(\tau_{p \mathrm{~d}}, \tau_{q \mathrm{~d}}\right)\right]_{i}$ are the variance-covariances matrix of pseudo-static and dynamic stresses at the $i$-th node; $\left[\operatorname{Cov}\left(\tau_{p \mathrm{~d}}, \tau_{q \mathrm{~s}}\right)\right]_{i}$ and $\left[\operatorname{Cov}\left(\tau_{p \mathrm{~s}}, \tau_{q \mathrm{~d}}\right)\right]_{i}$ are the variance-covariance matrix between static and dynamic stresses; and $p=$ $1, \ldots, 6$ and $q=1, \ldots, 6$ are subscripts denoting the stress components, in which the six stress components $\tau_{x}, \tau_{y}, \tau_{z}, \tau_{x y}, \tau_{x z}$ and $\tau_{y z}$ are denoted by $\tau_{1}, \tau_{2}, \tau_{3}, \tau_{4}, \tau_{5}$ and $\tau_{6}$, respectively. In expanded form, the variance-covariance matrix is

$$
\begin{aligned}
& {\left[\operatorname{Cov}\left(\tau_{p}, \tau_{q}\right)\right]_{i}} \\
& =\left[\begin{array}{cccc}
\operatorname{Var}\left(\tau_{1}\right) & \operatorname{Cov}\left(\tau_{1}, \tau_{2}\right) & \ldots & \operatorname{Cov}\left(\tau_{1}, \tau_{6}\right) \\
\operatorname{Cov}\left(\tau_{2}, \tau_{1}\right) & \operatorname{Var}\left(\tau_{2}\right) & \ldots & \operatorname{Cov}\left(\tau_{2}, \tau_{6}\right) \\
\ldots & \ldots & \ldots & \ldots \\
\operatorname{Cov}\left(\tau_{6}, \tau_{1}\right) & \operatorname{Cov}\left(\tau_{6}, \tau_{2}\right) \ldots & \operatorname{Var}\left(\tau_{6}\right)
\end{array}\right]_{i} .
\end{aligned}
$$

Note that $\left[\operatorname{Cov}\left(\tau_{p}, \tau_{q}\right)\right]_{i}$ is a symmetric matrix, thus, only 21 components need be solved for 3D problems. Similar to the displacement responses in Eqs (3), (4) and (5), the covariance of the dynamic stresses at the $i$-th node can be expressed as

$$
\begin{gathered}
{\left[\operatorname{Cov}\left(\tau_{p \mathrm{~d}}, \tau_{q \mathrm{~d}}\right)\right]_{i}=\sum_{k=1}^{n} \sum_{j=1}^{n} \frac{1}{2}\left(\Psi_{u j} \Psi_{\nu k}+\Psi_{\nu j} \Psi_{u k}\right) I_{j k},} \\
p, q=1, \ldots, 6, \quad i=1, \ldots, N_{n} .
\end{gathered}
$$

where $[\Psi]$ are the stress mode shapes, the subscripts $u$ and $\nu$ are $u=6(i-1)+p$ and $\nu=6(i-1)+q$, $N_{n}$ is the total number of nodes, $n$ is the number of modes used, and $I_{j k}$ represents the terms within the square brackets in Eq. (3). The pseudo-static stresses may be expressed as

$$
\left\{\tau_{\mathrm{s}}\right\}=[\eta]\left\{u_{\mathrm{R}}\right\}
$$

where the $j$-th column of $[\eta]$ are the stresses due to a unit displacement along the $j$-th restrained DOF. Consequently, the covariance matrix can be obtained as

$$
\begin{aligned}
& {\left[\operatorname{Cov}\left(\tau_{p \mathrm{~s}}, \tau_{q \mathrm{~s}}\right)\right]_{i}} \\
& =\sum_{l=1}^{r} \sum_{m=1}^{r} \frac{1}{2}\left(\eta_{u l} \eta_{\nu m}+\eta_{\nu l} \eta_{u m}\right) \\
& \quad \times \int_{-\infty}^{\infty} \frac{1}{\omega^{4}} S_{\ddot{u}_{\mathrm{R}_{l}} \ddot{u}_{\mathrm{R}_{m}}}(\omega) \mathrm{d} \omega \\
& \quad p, q=1, \ldots, 6, \quad i=1, \ldots, N_{n} .
\end{aligned}
$$

Finally, the covariance matrix between the pseudostatic and dynamic stress responses at the $i$-th node can be expressed as

$$
\begin{aligned}
& {\left[\operatorname{Cov}\left(\tau_{p \mathrm{~d}}, \tau_{q \mathrm{~s}}\right)+\operatorname{Cov}\left(\tau_{p \mathrm{~s}}, \tau_{q \mathrm{~d}}\right)\right]_{i}} \\
& =\operatorname{Re}\left[\sum_{j=1}^{n} \sum_{l=1}^{r} \sum_{m=1}^{r}\left(\Psi_{u j} \eta_{\nu l}+\Psi_{\nu j} \eta_{u l}\right)\right. \\
& \left.\quad \times \int_{-\infty}^{\infty} \Gamma_{m j} \frac{-1}{\omega^{2}} S_{\ddot{u}_{\mathrm{R}_{l}} \ddot{u}_{\mathrm{R}_{m}}}(\omega) \mathrm{d} \omega\right], \\
& p, q=1, \ldots, 6, \quad i=1, \ldots, N_{n},
\end{aligned}
$$

where $\operatorname{Re}[\cdot]$ denotes the real part.

\section{Von Mises stress simulation}

MCS is a well-known and straightforward method for estimating the statistics of a random variable, such as the von Mises stress $\tau_{\text {eq. Numerous samples of }}$ Gaussian Cartesian stresses, consistent with the covariance matrix computed through RVA, are first simulated at each node. Morris [11] provides a convenient subroutine to generate random multivariate normal samples with a specified mean and covariance matrix. The density function of the multivariate Gaussian distribution for $\{\tau\}=\left[\tau_{1}, \ldots, \tau_{m}\right]^{\mathrm{T}}$ is given by

$$
\begin{gathered}
f(\tau)=\frac{1}{\sqrt{(2 \pi)^{m} \operatorname{det}([C])}} \\
\times \exp \left(-\frac{1}{2}(\{\tau\}-\{\mu\})[C]^{-1}\right. \\
\left.\quad \times(\{\tau\}-\{\mu\})^{\mathrm{T}}\right),
\end{gathered}
$$


in which $[C]$ is the covariance matrix of the variates $\{\tau\}$ and $\{\mu\}$ is the mean vector. In the case studied, $\{\mu\}=\{0\}$ and $m=6$ for 3D problems.

Samples of $\tau_{\text {eq }}$ based on von Mises theory (Boresi and Sidebottom [3]) are determined from each set of Cartesian stresses through

$$
\begin{aligned}
\tau_{\mathrm{eq}}= & {\left[\frac{\left(\tau_{x x}-\tau_{y y}\right)^{2}+\left(\tau_{x x}-\tau_{z z}\right)^{2}+\left(\tau_{y y}-\tau_{z z}\right)^{2}}{2}\right.} \\
& \left.+3\left(\tau_{x y}^{2}+\tau_{x z}^{2}+\tau_{y z}^{2}\right)\right]^{1 / 2}
\end{aligned}
$$

A sufficient number of samples must be generated to obtain acceptable accuracy in the computed statistics. About 10000 samples was found to be adequate to perform scatter analysis for the mean $(\mu)$, standard deviation $(\sigma)$, skewness coefficient $\left(C_{\mathrm{s}}\right)$ and kurtosis coefficient $\left(C_{\mathrm{k}}\right)$ of $\tau_{\mathrm{eq}}$, in which (Harr [9])

$$
\begin{aligned}
C_{\mathrm{s}} & =\frac{\sum_{i=1}\left(\left(\tau_{i}\right)_{\mathrm{eq}}-\mu_{\tau_{\mathrm{eq}}}\right)^{3}}{N \sigma_{\tau_{\mathrm{eq}}}^{3}} \text { and } \\
C_{\mathrm{k}} & =\frac{\sum_{i=1}\left(\left(\tau_{i}\right)_{\mathrm{eq}}-\mu_{\tau_{\mathrm{eq}}}\right)^{4}}{N \sigma_{\tau_{\mathrm{eq}}}^{4}},
\end{aligned}
$$

where $N$ is the total number of simulated values.

In failure analysis, a deterministic or random design strength, $\tau_{\mathrm{eq}}^{*}$, may be specified and statistics of the safety margin, $M=\tau_{\text {eq }}^{*}-\tau_{\text {eq }}$, can be computed. In this study, $\tau_{\text {eq }}^{*}$ and $\tau_{\text {eq }}$ are assumed to be statistically independent. In general, a comprehensive reliability analysis should compute the Hasofer-Lind reliability index, which is invariant with respect to different but mechanically equivalent formulations of the failure criterion (Madsen et al. [10]). Here, because $M$ is a linear function of $\tau_{\text {eq }}^{*}$ and $\tau_{\text {eq }}$, the Hasofer-Lind reliability index is identical to the Cornell reliability index, $\mu_{M} / \sigma_{M}$, where $\mu_{M}$ and $\sigma_{M}$ are the mean and standard deviation of $M$. The required number of simulations $(N)$ for acceptable accuracy is dependent on the design failure probability, $p_{\mathrm{f}}$, with the rule-of-thumb being:

$$
N=\left\{\begin{aligned}
10000 & \text { if } 0.01<p_{\mathrm{f}}<1 \\
100000 & \text { if } 0.001<p_{\mathrm{f}} \leqslant 0.01 \\
1000000 & \text { if } 0.0001 \leqslant p_{\mathrm{f}} \leqslant 0.001
\end{aligned}\right.
$$

In general, $N \geqslant 100 / p_{\mathrm{f}}$. The probability of failure is determined by $p_{\mathrm{f}}=N_{\mathrm{f}} / N$ where $N_{\mathrm{f}}$ is the total number of failure events. For $N=1000000$, the sample generation takes only about 20 seconds on a SGI IRIS4D Unix workstation. For $p_{\mathrm{f}}<0.01 \%$, direct MCS is not recommended because of the very larger number of samples required. Alternatively, efficient MCS using importance sampling, the first order reliability method (FORM), or the second order reliability method (SORM) may be used (Ang and Tang [1]). In these methods the most probable failure point needs to be solved using an iterative optimization technique. On the other hand, for the many structures that are designed with $p_{\mathrm{f}}>0.01 \%$, the proposed technique is quick and simple.

The accuracy of the estimated probability of failure also can be estimated using the MCS results. As $N$ approaches infinity, $p_{\mathrm{f}}$ approaches the true value. Assume that each simulation cycle constitutes a Bernoulli trial, the variance of the estimated probability of failure can be computed approximately as (Ayyub and Mccuen [2])

$$
\operatorname{Var}\left(p_{\mathrm{f}}\right)=\frac{\left(1-p_{\mathrm{f}}\right) p_{\mathrm{f}}}{N} .
$$

Consequently, the accuracy of the estimated $p_{\mathrm{f}}$ can be measured by its coefficient of variation

$$
\operatorname{COV}\left(p_{\mathrm{f}}\right)=\frac{1}{p_{\mathrm{f}}} \sqrt{\frac{\left(1-p_{\mathrm{f}}\right) p_{\mathrm{f}}}{N}} .
$$

The smaller the coefficient of variation, the better the accuracy of the estimated $p_{\mathrm{f}}$. Equation (18) always yields a coefficient of variation of less than $10 \%$, which is acceptable for most engineering problems.

Another feature of the proposed design technique is that the design strength, $\tau_{\mathrm{eq}}^{*}$, corresponding to a specified probability of failure can be computed. The result can be cross-examined with that in failure analysis. The array of $N$ simulated samples are first sorted in decreasing order as $x_{1} \geqslant x_{2} \geqslant \cdots \geqslant x_{N}$. The design strength corresponding to a probability of failure $p_{\mathrm{f}}$ is then the value of the $i$-th sample, where $i=$ integer part of $N p_{\mathrm{f}}$. For very small $p_{\mathrm{f}}$, the arrangement of the large number of simulated samples in decreasing order would be very time-consuming and inefficient. An improved technique is to use a threshold stress, $\tau_{\mathrm{T}}$, to filter out the larger values of $\tau_{\mathrm{eq}}$ which could possibly cause failure, and then rank order only the filtered values. A suitable threshold is

$$
\begin{aligned}
\tau_{\mathrm{T}} & =0.8\left(\widehat{\mu}+\left(2.3+0.71 \log _{10}\left(\frac{0.01}{p_{\mathrm{f}}}\right)+C_{\mathrm{s}}\right) \widehat{\sigma}\right) \\
& \geqslant \widehat{\mu}, \\
& 0.01 \leqslant p_{\mathrm{f}} \leqslant 0.0001,
\end{aligned}
$$

in which $\widehat{\mu}$ and $\widehat{\sigma}$ are the mean and standard deviation of $\tau_{\text {eq }}$ estimated from a small number of $\tau_{\text {eq }}$ samples (say 2000 values). The coefficient of 0.8 is used to ensure that no probable failure samples are left out due to estimation errors in $\widehat{\mu}$ and $\widehat{\sigma}$. 


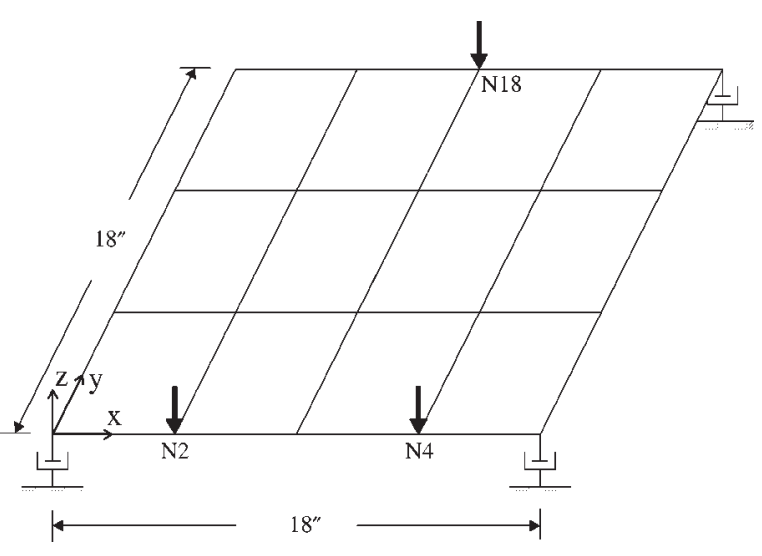

Fig. 1. Mesh and boundary conditions for shaker table.

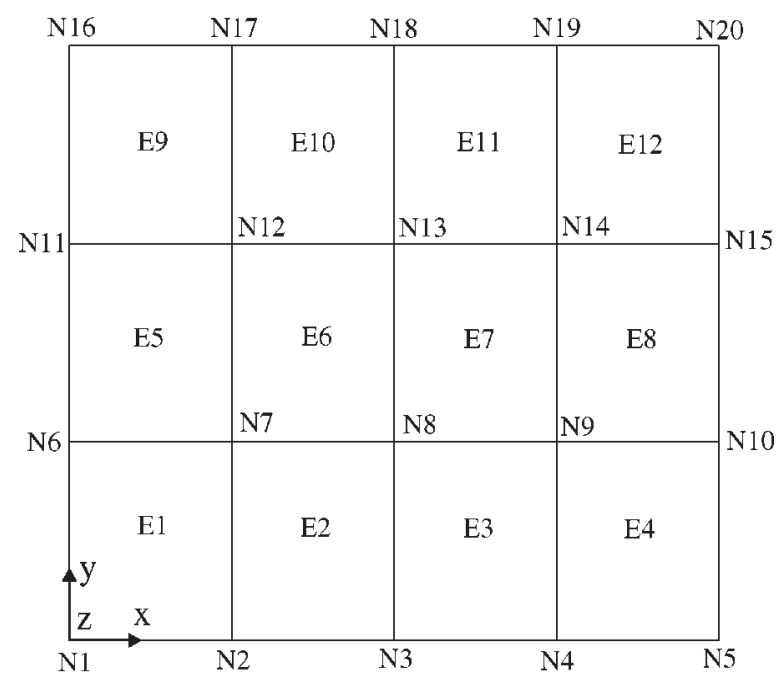

Fig. 2. Node and element numbers.

\section{Example}

An aluminum shaker table with a spring-damper for each leg is used to illustrate the proposed technique. The structure having a dimension of $0.457 \mathrm{~m} \times$ $0.457 \mathrm{~m}\left(18^{\prime \prime} \times 18^{\prime \prime}\right)$ is represented by a finite element model as shown in Fig. 1. The model consists of 12 elastic shell elements (SHELL 63) and 4 spring-dampers (COMBINE 14). The four nodes at the base of the dampers are completely restrained and two of the nodes connecting the table and the dampers (nodes 1 and 5) are restrained in the $X$ and $Y$ directions. The node and element labels are given in Fig. 2. Random forces with the PSD shown in Fig. 3(a) are applied in the $Z$ direction at nodes 2 and 4. Another random force with the PSD shown in Fig. 3(b) is applied in the $Z$ direction at node 18 .

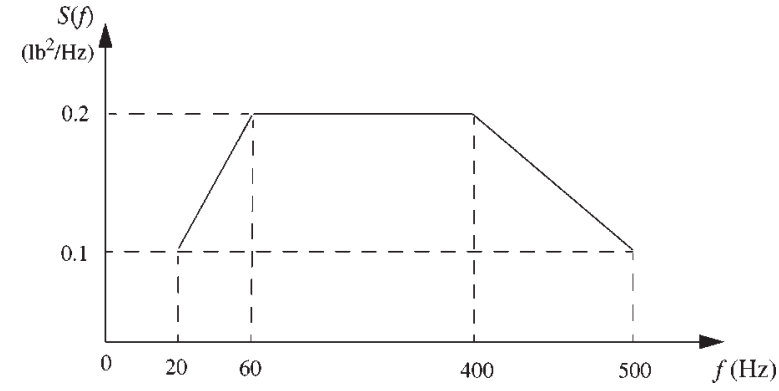

(a)

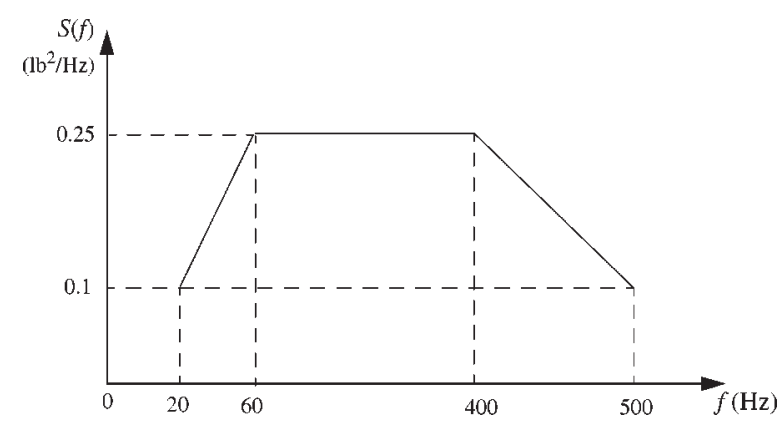

(b)

Fig. 3. Power spectral density function for out-of-plane excitations at: (a) nodes 2 and 4, and (b) node 18 .

The two sets of forces are assumed to be statistically independent. Root-mean-square values of the nodal stresses, $\tau_{x}, \tau_{y}$ and $\tau_{x y}$, for each element are shown in Figs 4, 5 and 6, respectively. The largest responses in the figures are $37.2 \mathrm{MPa}(5399 \mathrm{psi})$ for $\tau_{x}, 35.7 \mathrm{MPa}$ (5177 psi) for $\tau_{y}$ and $10.6 \mathrm{MPa}(1533 \mathrm{psi})$ for $\tau_{x y}$, which occur at nodes 3,18 and 15 , respectively. The contours are plotted without averaging the responses on the boundary of each element.

In order to obtain the maximum von Mises stress for design, covariance matrices were computed for some critical points which had large Cartesian stresses. Since only $\tau_{x}, \tau_{y}$ and $\tau_{x y}$ are available for the shell elements, only six elements of the covariance matrices are required for computing the von Mises stress, and the results are given in Table 1. The mean, standard deviation, skewness coefficient and kurtosis coefficient of the von Mises stress response obtained via MCS are listed in Table 2. It can be seen that node 3 has a maximum mean value of $33.9 \mathrm{MPa}(4910 \mathrm{psi})$ and a maximum standard deviation of $17.6 \mathrm{MPa}$ (2545 psi). Histograms for the PDF and CDF of the stress at node 3 are shown in Fig. 7 (a) and (b), respectively. Note that the von Mises stress response has a non-zero mean and is 

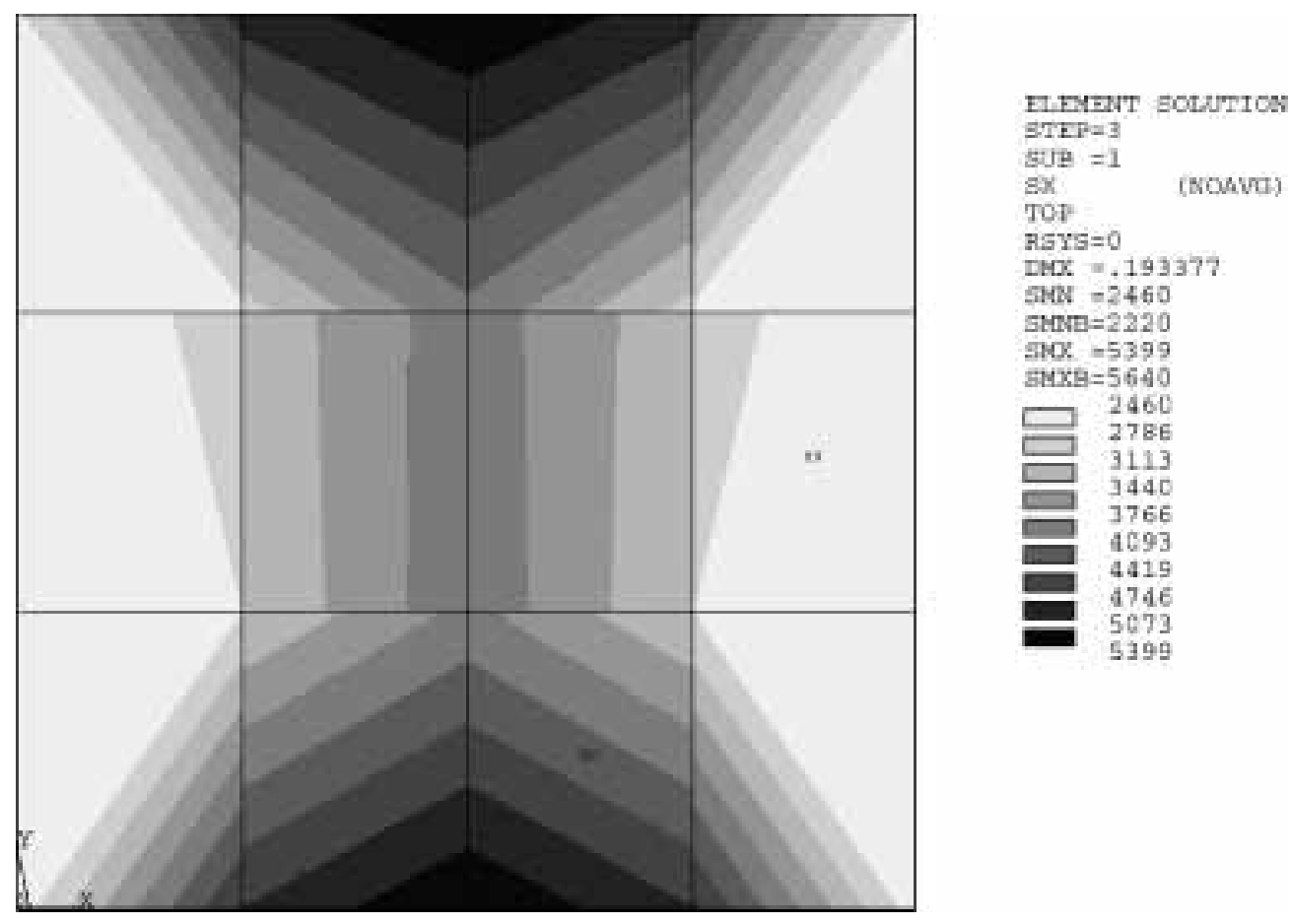

Fig. 4. Contours of root-mean-square response of $X$ normal stress component.

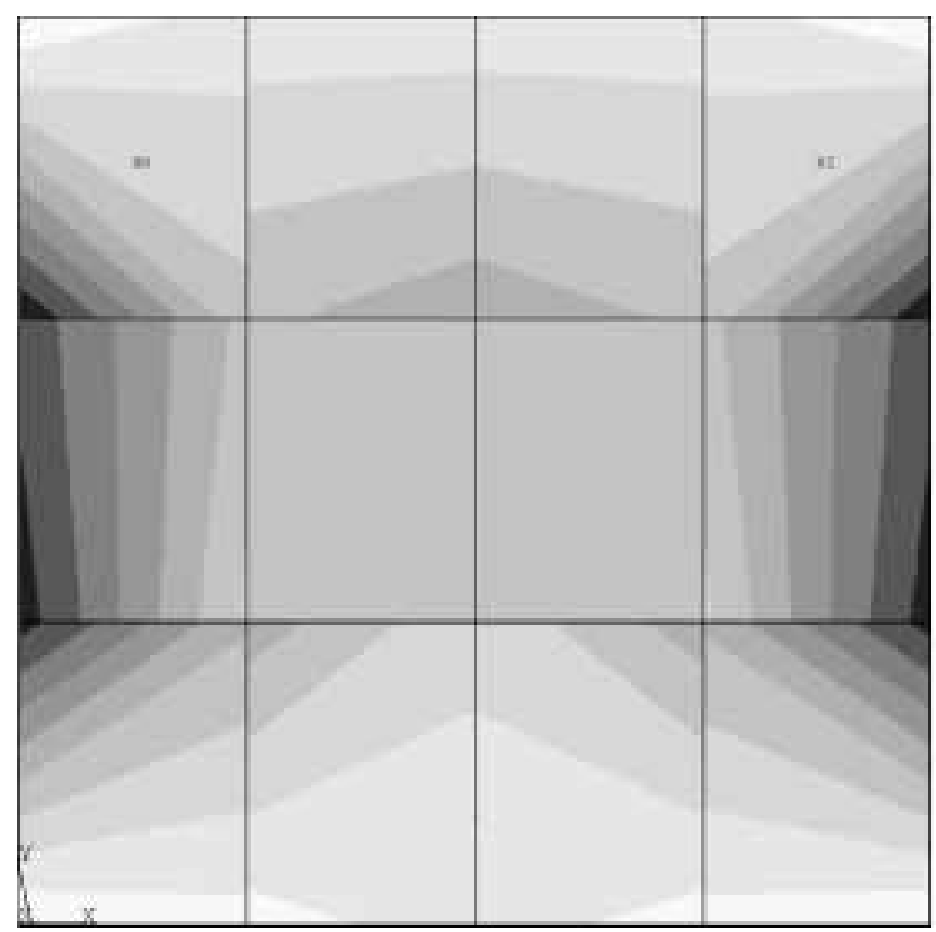

ELEMEIT BOLUTIOA gTEP=3 sty $=1$ BY (Noavo) TOP

RGY IMX $=.19337 \%$ Sim -2305 Bसte $=2562$ $\sin x-5177$

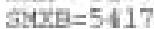
2805
-3056 $\square \begin{array}{r}2805 \\ 3065\end{array}$ $\Longrightarrow 3332$ 3596 3859

4123
4386

$-4650$

4914
5179

Fig. 5. Contours of root-mean-square response of $Y$ normal stress component. 

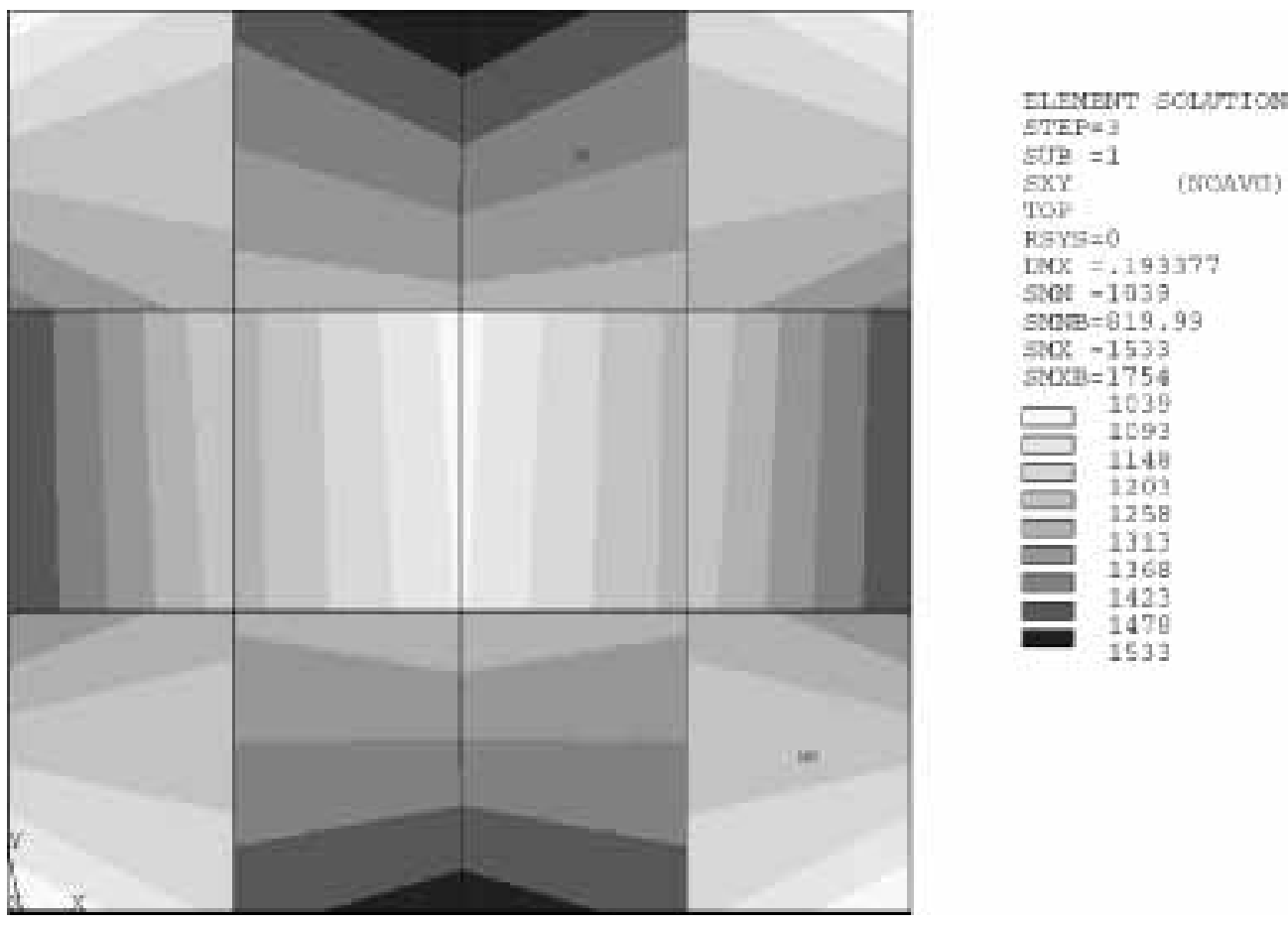

Fig. 6. Contours of root-mean-square response of $X Y$ shear stress component.

Table 1

Variance-covariance components of Cartesian stresses at the critical points $\mathrm{MPa}^{2}\left(\mathrm{lb}^{2} / \mathrm{in}^{4}\right)$

\begin{tabular}{ccccc}
\hline $\begin{array}{c}\text { Components } \\
\text { of var-covar. } \\
\text { matrix }\end{array}$ & $\begin{array}{c}\text { Node } 3 \\
\text { of } \\
\text { element } 3\end{array}$ & $\begin{array}{c}\text { Node } 18 \\
\text { of } \\
\text { element } 11\end{array}$ & $\begin{array}{c}\text { Node } 15 \\
\text { of } \\
\text { element } 12\end{array}$ & $\begin{array}{c}\text { Node } 10 \\
\text { of } \\
\text { element } 8\end{array}$ \\
\hline $\operatorname{Var}\left(\tau_{x}\right)$ & $1386\left(2.92 \times 10^{7}\right)$ & $1369\left(2.88 \times 10^{7}\right)$ & $336\left(7.07 \times 10^{6}\right)$ & $318\left(6.68 \times 10^{6}\right)$ \\
$\operatorname{Cov}\left(\tau_{x}, \tau_{y}\right)$ & $729\left(1.53 \times 10^{7}\right)$ & $721\left(1.52 \times 10^{7}\right)$ & $596\left(1.25 \times 10^{7}\right)$ & $553\left(1.16 \times 10^{7}\right)$ \\
$\operatorname{Cov}\left(\tau_{x}, \tau_{x y}\right)$ & $-23.5\left(-5.0 \times 10^{5}\right)$ & $33.6\left(7.07 \times 10^{5}\right)$ & $1.15\left(2.42 \times 10^{4}\right)$ & $49.9\left(1.05 \times 10^{6}\right)$ \\
$\operatorname{Var}\left(\tau_{y}\right)$ & $487\left(1.02 \times 10^{7}\right)$ & $480\left(1.01 \times 10^{7}\right)$ & $1274\left(2.68 \times 10^{7}\right)$ & $1221\left(2.57 \times 10^{7}\right)$ \\
$\operatorname{Cov}\left(\tau_{y}, \tau_{x y}\right)$ & $-12.8\left(-2.7 \times 10^{5}\right)$ & $19.6\left(4.12 \times 10^{5}\right)$ & $-8.89\left(-1.9 \times 10^{5}\right)$ & $94.1\left(1.98 \times 10^{6}\right)$ \\
$\operatorname{Var}\left(\tau_{x y}\right)$ & $109\left(2.29 \times 10^{6}\right)$ & $112\left(2.35 \times 10^{6}\right)$ & $92.6\left(1.95 \times 10^{6}\right)$ & $104\left(2.19 \times 10^{6}\right)$ \\
\hline
\end{tabular}

Table 2

Statistics of von Mises stress at the critical points

\begin{tabular}{ccccc}
\hline Statistic & $\begin{array}{c}\text { Node 3 of } \\
\text { element 3 }\end{array}$ & $\begin{array}{c}\text { Node 18 of } \\
\text { element 11 }\end{array}$ & $\begin{array}{c}\text { Node 15 of } \\
\text { element 12 }\end{array}$ & $\begin{array}{c}\text { Node 10 of } \\
\text { element } 8\end{array}$ \\
\hline Mean $(\mathrm{MPa})$ & 33.86 & 33.78 & 31.52 & 31.68 \\
$(\mathrm{psi})$ & $(4910.4)$ & $(4898.6)$ & $(4570.6)$ & $(4593.8)$ \\
Standard deviation $(\mathrm{MPa})$ & 17.55 & 17.49 & 17.06 & 16.95 \\
$(\mathrm{psi})$ & $(2545.2)$ & $(2536.0)$ & $(2474.2)$ & $(2457.8)$ \\
Skewness coefficient & 0.985 & 0.981 & 1.012 & 1.004 \\
Kurtosis coefficient & 4.174 & 4.173 & 4.253 & 4.266 \\
\hline
\end{tabular}




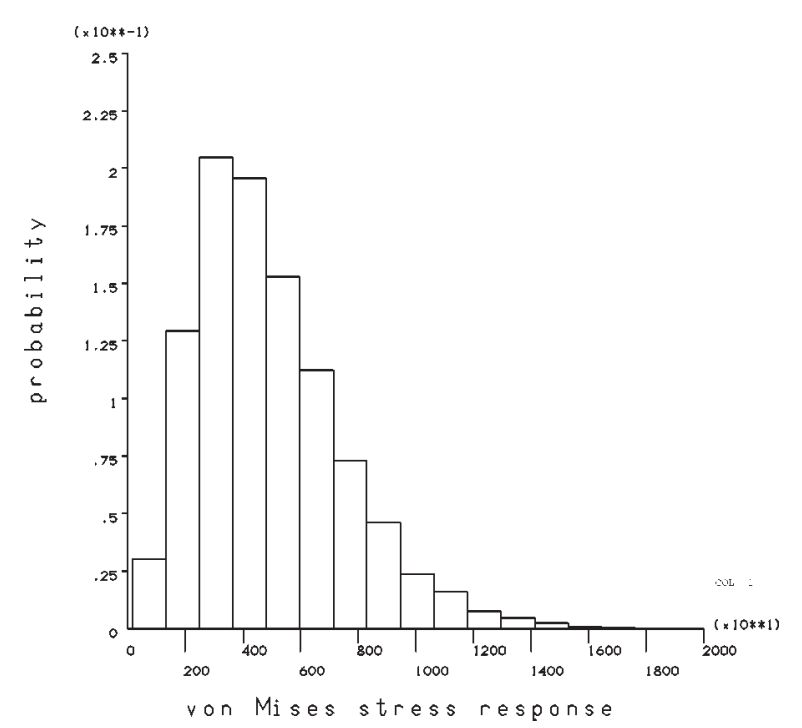

(a)

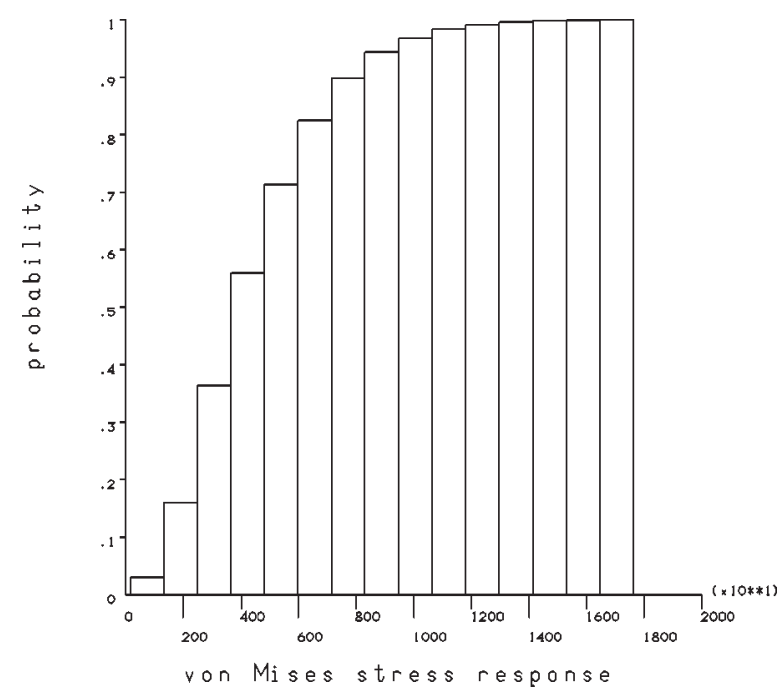

(b)

Fig. 7. Histograms for (a) probability density function and (b) cumulative distribution function of von Mises stress response at node 3 .

non-Gaussian even though the excitations are zeromean Gaussian processes. For a trial failure probability of $p_{\mathrm{f}}=0.005$, the proposed technique gives the required design strength as approximately 93.4 $\mathrm{MPa}$ (13540 psi). Using a design strength of $93.4 \mathrm{MPa}$ (13540 psi) and assuming a coefficient of variation of $10 \%$ for this strength due to manufacturing variations, failure analysis yields $p_{\mathrm{f}}=0.0074$ with a coefficient of variation of $3.7 \%$. Therefore, based on a $95 \%$ confidence level, $p_{\mathrm{f}}$ will be less than 0.00786 , i.e., the shaker table will have a reliability of 0.992 under random excitations.

\section{Summary}

In recent years, random vibration analysis has become available in several commercial finite element software for computing the statistics of Cartesian responses, such as displacements, stresses and strains. Reliability-based design of structures subject to random excitations is desirable for a variety of engineering problems, e.g., fatigue, yield and noise control. However, Cartesian responses are often inadequate for expressing realistic structural design criteria. For ductile material, the von Mises (or distortion energy) theory, which is based on a single equivalent stress response, is preferred. Statistics of the von Mises stress response are computed by means of Monte Carlo simulation (MCS) using the $6 \times 6$ variance-covariance matrix of Cartesian stresses at each node computed through random vibration analysis. Consequently, the scatter of the von Mises stresses due to random excitations can be quantified. The probability of failure corresponding to a specified design strength can be estimated enabling the reliability-based design of structures subjected to random excitations. In addition, the required design strength corresponding to a target failure probability can also be estimated yielding useful information for manufacturing. A simple example consisting of a shaker table subject to random vibrations is used to demonstrate the advantages of the proposed techniques.

The combination of random vibration analysis and MCS yields a powerful yet simple method for statistical analysis. Since the von Mises stress can be related to Cartesian stresses in closed-form, the MCS is very efficient. It should be noted that the proposed technique is also applicable to other measures used in failure analysis, such as the stress intensity factor in fracture mechanics, the maximum shear stress for soil mechanics problems, etc. In practice, engineering structures are usually designed with reliabilities ranging from $95 \%$ to $99.99 \%$ and the required number of simulations is feasible using engineering workstations. On the other hand, advanced probabilistic analysis methods, such as efficient MCS, FORM and SORM, need to solve for the most probable failure point using a nonlinear optimization technique which offsets the computational efficiency of these methods. 


\section{References}

[1] A.H.-S. Ang and W.H. Tang, Probability Concepts in Engineering Planning and Design, Vol. II, Wiley, New York, 1984, pp. 333-447.

[2] B.M. Ayyub and R.H. Mccuen, Simulation-based reliability methods, in: Probabilistic Structural Mechanics Handbook, C. Sundararajam, ed., Chapman and Hall, New York, 1995, pp. 53-69.

[3] A.P. Boresi and O.M. Sidebottom, Advanced Mechanics of Materials, Wiley, New York, 1985

[4] M.-T. Chen, Response of an Earth Dam to Spatially Varying Earthquake Ground Motion, $\mathrm{PhD}$ dissertation, Michigan State University, 1995, pp. 94-148.

[5] M.-T. Chen and A. Ali, A robust integration technique for general-purpose random vibration analysis, in: Proc. of the 67th Shock and Vibration Symposium, Monterey, California, 1996, pp. 479-488.

[6] R.S. Harichandran, Random vibration under propagating excitation: Closed-form solutions, J. Eng. Mech. 118(3) (1992), 575-586.
[7] R.S. Harichandran and M.-T. Chen, Statistical moments of principal stress-related quantities in random vibration analysis, in: Proc. 7th ASCE Specialty Conference on Probabilistic Mechanics and Structural Reliability, Worcester, MA, 1996, pp. 962-965.

[8] R.S. Harichandran and W. Wang, Response of indeterminate two-span beam to spatially varying seismic excitation, Earthquake Eng. Struct. Dynamics 19(2) (1990), 173-187.

[9] M.E. Harr, Reliability-Based Design in Civil Engineering, McGraw-Hill, New York, 1987.

[10] H.O. Madsen, S. Krenk and N.C. Lind, Method of Structural Safety, Prentice-Hall, Englewood Cliffs, NJ, 1986.

[11] A.H. Morris, NSWC library of mathematics subroutines, Report No. NSWCDD/TR-92/425, Naval Surface Warfare Center, Dahlgren, VA, 1993.

[12] N.C. Nigam, Applications of Random Vibrations, SpringerVerlag, New York, 1994

[13] T.T. Soong and M. Grigoriu, Random Vibration of Mechanical and Structural Systems, Prentice-Hall, Englewood Cliffs, NJ, 1993.

Received 29 January 1997; Revised 8 December 1997 

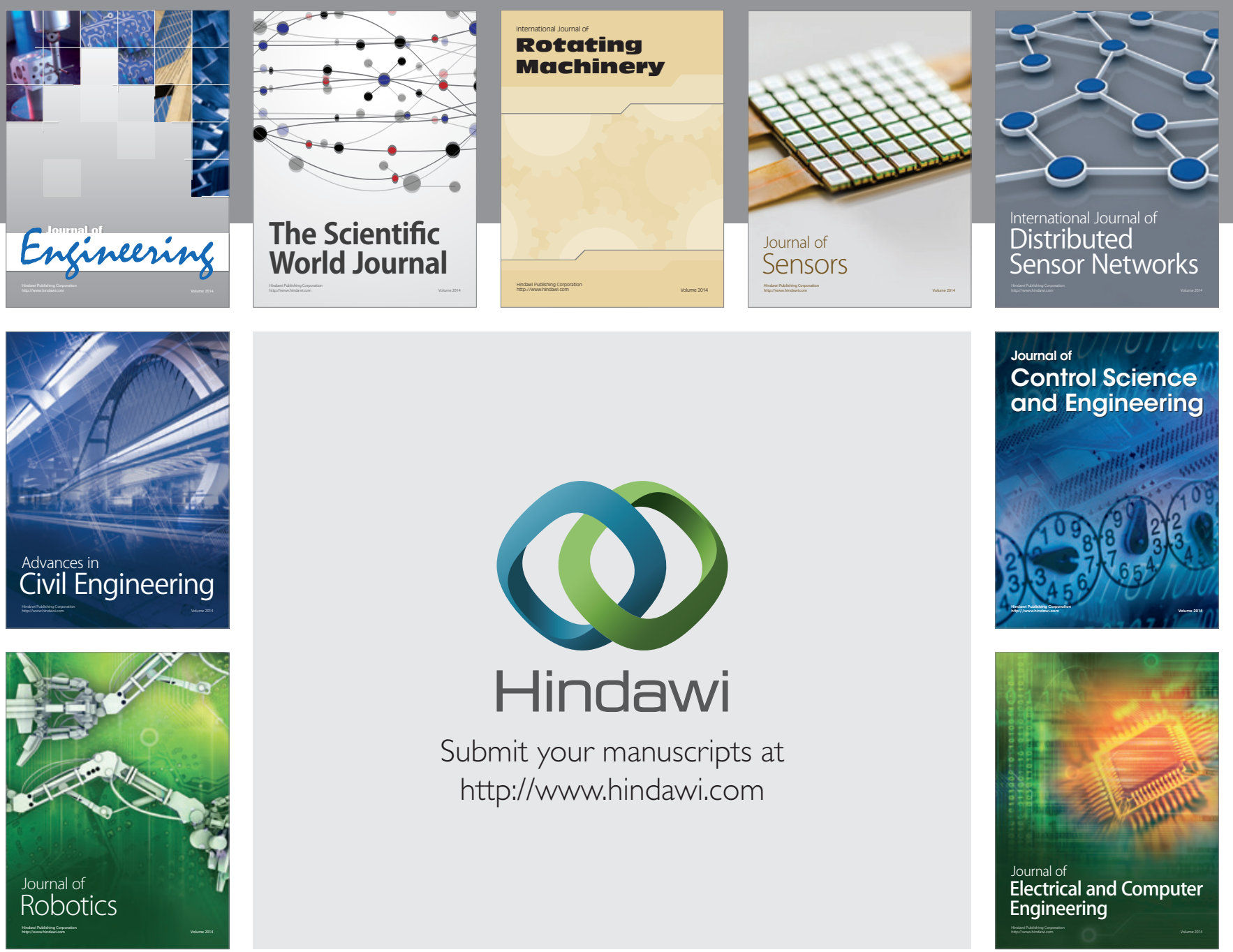

Submit your manuscripts at

http://www.hindawi.com
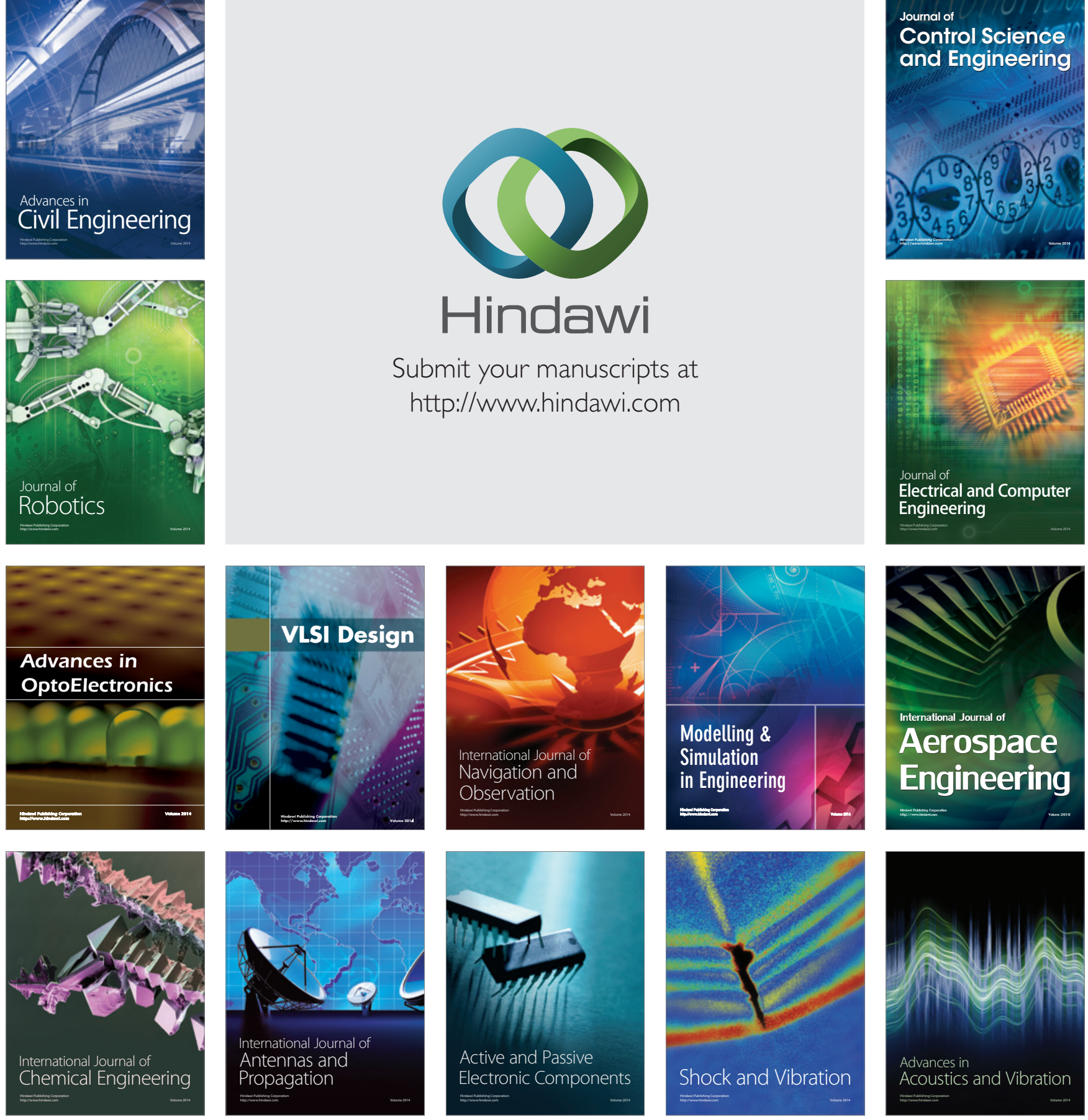